\title{
Design of the commissioning filter/mask/window assembly for undulator beamline front ends at the Advanced Photon Source
}

Deming Shu and Tuncer M. Kuzay
Experimental Facilities Division, Advanced Photon Source, Argonne National Laboratory, Argonne. IL
60439

(Presented on October 20, 1995)

A compact filter/mask/window assembly has been designed for undulator beamline commissioning activity at the Advanced Photon Source beamlines. The assembly consists of one $300-\mu \mathrm{m}$ graphite filter, one $127-\mu \mathrm{m}$ CVD diamond filter and two $250-\mu \mathrm{m}$ beryllium windows. A water-cooled Glidcop fixed mask with a $4.5-\mathrm{mm}$ X $4.5-\mathrm{mm}$ output optical aperture and a $0.96-\mathrm{mrad}$ $\mathrm{X} 1.6-\mathrm{mrad}$ beam missteering acceptance is a major part in the assembly. The CVD diamond filter which is mounted on the downstream side of the fixed mask is designed to also function as a transmitting $\mathrm{x}$-ray beam position monitor. The sum signal from the latter can be used to monitor the physical condition of the graphite filter and prevent any possible chain reaction damage to the beryllium windows downstream. In this paper, the design concept as well as the detailed structural design of the commisioning window are presented. Further applications of the commissioning window components are also discussed.C 1995 American Institute of Physics

\section{INTRODUCTION}

Undulator A for the Advanced Photon Source (APS) is an insertion device that will generate high-intensity $\mathrm{X}$-ray radiation in the spectral range $3.2 \mathrm{keV}$ to $45 \mathrm{keV}$ by using the first, third, and fifth harmonics of radiation. $6 \mathrm{~kW}$ of total emitted power with $160 \mathrm{~W} / \mathrm{mm} 2$ peak heat flux is specified at $30-\mathrm{m}$ from the source when the undulator gap is closed'. A windowless front-end with a differential pumping system has been designed as a standard undulator/wiggler compatible configuration for the APS undulator $A^{2,3}$. However, it is recognized that, during the commissioning period, a reliable vacuum window system with a water-cooled fixed mask will give more flexibility to the user in beamline commissioning activities. The vacuum windows will allow users to relax the beamline vacuum requirements and save the vacuum preparation time to change the beamline configuration, which usually happens frequently during the commissioning period. The fixed mask in the assembly minimizes the undulator beam missteering range and simplifies the design of any downstream fixed mask on a beamline. Furthermore, the novel transmitting X-ray beam position monitor (TBPM) ${ }^{4}$ design makes it possible to integrate the BPM function with filter and/or window functions in a single unit in the commissioning window assembly to verify the beam position and monitor the upstream filter condition.

In this paper, the design concept as well as the detailed structural design of the commisioning are presented. Further applications of the commissioning window components are also discussed.

\section{DESIGN}

There were many restrictions affecting the design of the commissioning window. First, the space for the commissioning window was limited by the existing standand front-end design. Second. the total length of the commissioning window system should not exceed the total length of the APS differential pumping system that it replaces, which is only about $708 \mathrm{~mm}$ long. Furthermore, it is also preferred to design the window system to be compatible with the existing front-end vacuum connecting flanges and supporting tables. All of which meant a very compact design for the commissioning window assembly.

Safety and reliability are the major guides for the design of the commissioning window. Experimental data for the filter and window design in such a high heat flux and/or total power range are scarce. Hence a flexible modular design was preferred. The modular design will allow us to change the filter foil thickness and/or the number of the components to suit different experimental requirements. Design is based on the results of many extensive analytic studies using ANSYS ${ }^{5-7}$. A prototype is planned to be tested starting from a conservative filter thickness setting with limited beam load, which will then be optimized with the experimental data.

As one scenario, it can be hypothesiszed that because of the imperfections of the synthetic filter material, it is possible to have local damage on the first filter, which may cause a chain reaction in the downstream windows. An online monitor has been designed using the principles of a transmitting X-BPM on the second filter (i.e., the CVD diamond disk). The sum signal of the BPM will be monitored to prevent such an incident.

As shown in Fig. 1, the APS commissioning window assembly consists of four major modular components: a compact water-cooled filter (1), a water-cooled fixed mask with enhanced heat transfer (2), and two watercooled beryllium windows (3-4). A CVD diamond filter (5) is mounted on the downstream side of the fixed mask, which also serves as a transmitting $\mathrm{x}$-ray beam position monitor. An adjustable table-top supporting structure (6) provides compatibility with the existing differential-pump supporting table (7). To avoid the extra atmospheric shock wave on the filter, manual valves are designed to control the vacuum connection during the system pumping-down process. Two 
$11 \mathrm{l} / \mathrm{s}$ ion pumps (8) and two $2 \mathrm{l} / \mathrm{s}$ ion pumps (9) are installed on the system.

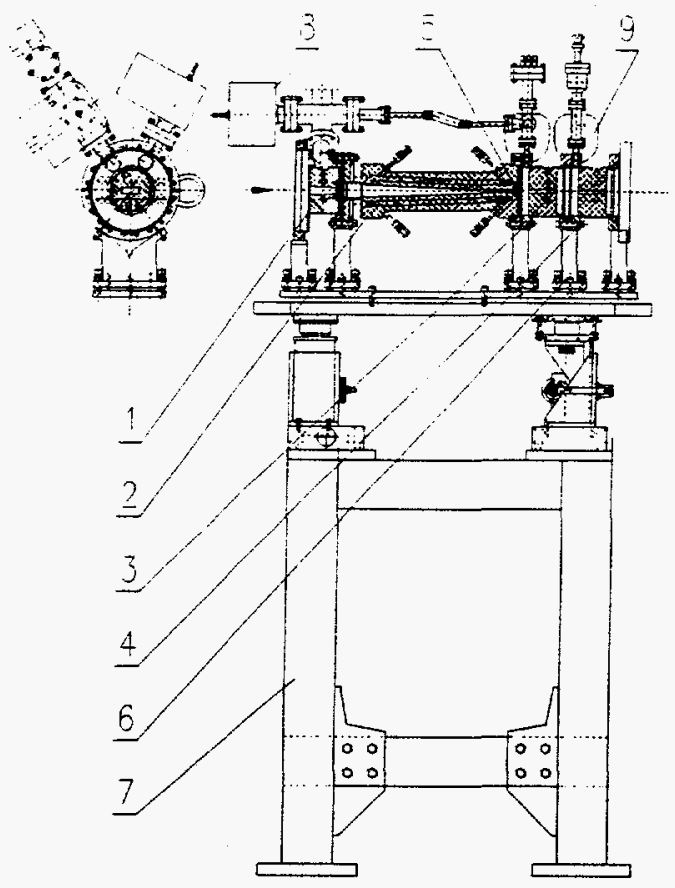

FIG.1. The APS commissioning window assembly, (1) compact water-cooled filter, (2) water-cooled fixed mask, (3-4) two watercooled beryllium windows, (5) CVD diamond filter, (6) adjustable table-top supporting structure, (7) supporting table, (8) $11 \mathrm{~V} / \mathrm{s}$ ion pumps, (9) $2 \mathrm{l} / \mathrm{s}$ ion pumps

Fig. 2 shows the commissioning window optical apertures. The $4.5-\mathrm{mm} \times 4.5-\mathrm{mm}$ fixed mask aperture size was determined by two major considerations. First, during the first year of the storage-ring commissioning period when the particle beam orbit may need to be adjusted locally for global performance optimization, a larger front-end exit optical aperture will tolerate more beam position change without realignment to the frontend. Second, $t$ he optical aperture should be large enough for the undulator performance diagnostic experiment. The aperture size of the first filter was designed to accept the maximum undulator beam missteering. For the two beryllium windows, compared with the fixed mask, $0.5-\mathrm{mm}$ and $1-\mathrm{mm}$ extra aperture size was added on the vertical aperture and $0.5-\mathrm{mm}$ and $3.5-\mathrm{mm}$ on the horizontal aperture to compensate for the flange-flange alignment tolerances, respectively, for the first and the second unit. Because of the limitations on the total length of the assembly, there are no bellows in-between the modular components.

As a starting point for the prototype test, the commissioning window system will contain one $300-\mu \mathrm{m}$ graphite filter, one 127- $\mu \mathrm{m}$ CVD diamond filter with a $0.2-$ $\mu \mathrm{m}$ aluminum coating, and two $250-\mu \mathrm{m}$ beryllium windows. Fig. 3 shows the $\mathrm{X}$-ray transmission percentage with this assembly at various photon energies.

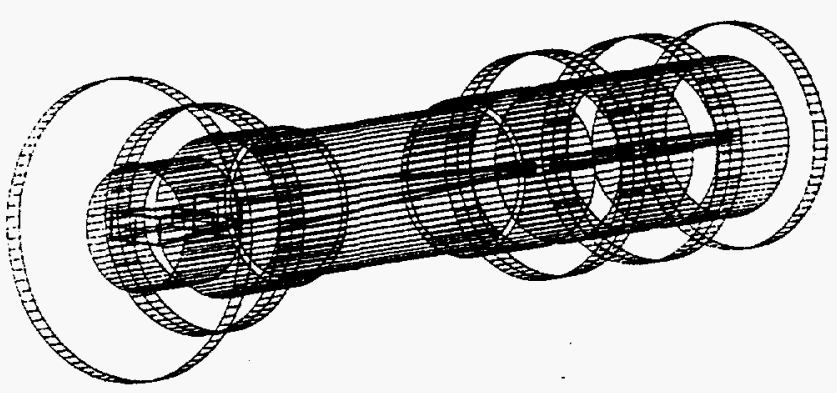

FIG.2. The commissioning window optical apertures

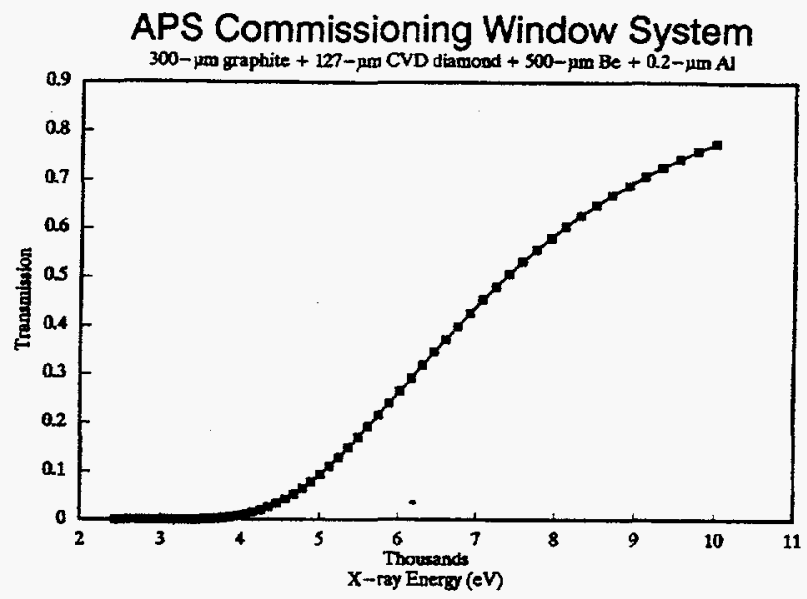

FIG.3. The X-ray transmission percentage with this assembly at various photon energies.

\section{COMPACT FILTER ASSEMBLY}

The compact filter assembly for the commissioning window consists of two parts: the main body weldment and the filter mounting structure (Fig.4). The water-cooled main body (1) is made of Glidcop. Two stainless steel flanges (2) are connected to the main body with an ultra-high-vacuum (UHV) tight explosive bonding joint ${ }^{8}$. The filter foil (3), made of graphite typically, is mounted to the main body by a $35-\mathrm{mm} \times 47-\mathrm{mm}$ spring loading clamping structure (4). The spring loading force is adjustable to optimize the water cooling efficiency and the boundary stress condition.

For a multi-foil filter application, the clamping plate can be replaced by a foil cooling magazine. The cooling magazine also provides the flexibility to change the filter vertical aperture. The minimum filter vertical optical aperture is $10-\mathrm{mm}$, which is determined by the maximum beam missteering ray tracing. 


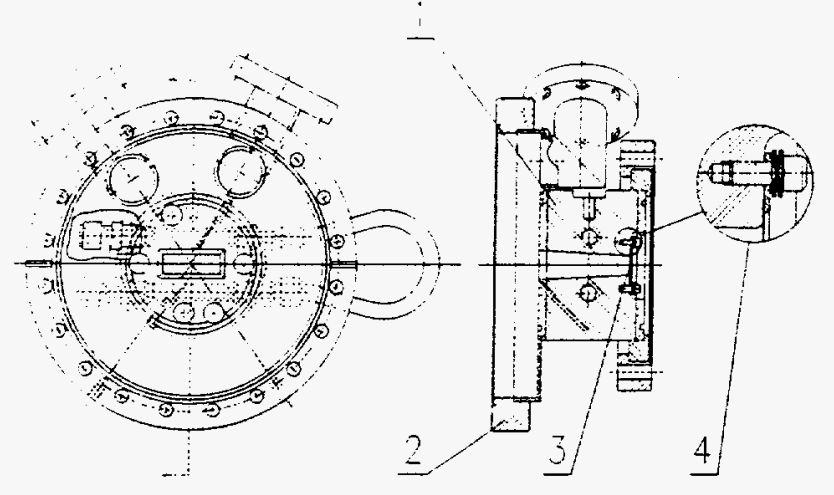

FIG.4. The compact filter assembly, (1) water-cooled main body, (2) stainless steel flanges (3) filter foil, (4) spring loading clamping structure

\section{FIXED MASK}

The fixed mask for the commissioning window is similar to the fixed mask in the APS frontend". The boxcone-shaped main body was made of Glidcop. An enhanced heat transfer mechanism, which increases the convective heat transfer coefficient to about $3 \mathrm{~W} / \mathrm{cm}^{2}$ with water as coolant, has been built in the main body. Explosive bonding was used to form the UHV joint between the main body and the stainless steel UHV flanges. As shown in Fig. 5, the fixed mask has a $23.7-\mathrm{mm} \mathrm{X} 40.2-\mathrm{mm}$ input aperture and a 4.5$\mathrm{mm}$ X 4.5-mm output optical aperture with a $0.96-\mathrm{mrad} X$ 1.6-mrad beam missteering acceptance. To withstand the powerful undulator A beam (up to $6 \mathrm{kw}$ total power without filtering ), a 2-degree vertical grazing incidence angle and a 4 degree horizontal grazing incidence angle have been specified in the fixed mask cooling surfaces, respectively.

By the downstream end of the main body, a special in-vacuum mounting surface has been prepared for the CVD diamond filter use. A precision survey plane with two fiducial holes is manufactured on the outside of the main body for alignment purposes.

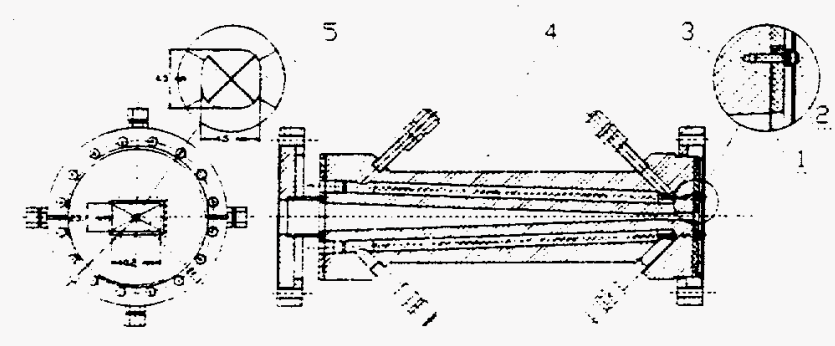

FIG.5. The fixed mask and filter/transmitting XBPM, (1) CVDdiamond disk. (2) spring-loaded clamping structure, (3) terminal interface film. (4) fixed mask main body, (5) four electronically isolated aluminum quadrant coating patterns.

\section{TRANSMITTING BEAM POSITION MONITOR (TBPM)}

The CVD diamond filter, which is a 25.4-mm diameter disk mounted on the downstream side of the fixed mask, is also designed as a transmitting $\mathrm{x}$-ray beam position monitor for the commissioning window system. The basic concept of the TBPM is to mount the monitor blade perpendicular to the synchrotron radiation beam and design the blade and its coating thicknesses in such a way that most of the X-ray beam will be transmitted through the blade (just like a filter or window). As shown in Fig. 5, the CVDdiamond disk (1) is coated with four electronically isolated aluminum quadrant patterns (2). The thickness of the aluminum coating is about $0.2-\mu \mathrm{m}$. The photoelectronemission signal will be collected by a terminal interface film (3), which is made from thin alumina film and is coated with gold. A spring-loaded clamping structure, which is similar to the one mounted on the compact filter assembly, provides an adjustable clamping force at the CVD diamond cooling interface. This design concept provides the possibility of integrating the filter with TBPM functions. In addition, the sum signal of the TBPM will be monitored to prevent any chain reaction incident caused by possible local failure sites on the first filter foil.

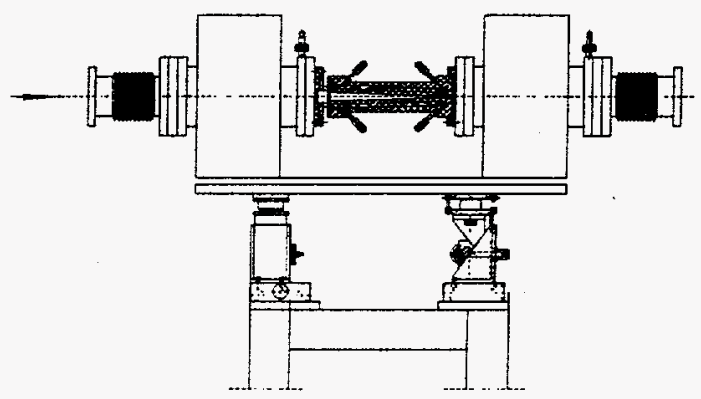

FIG.6. The same fixed mask in the commissioning window system can be assembled inbetween the two ion pumps as a part of the front-end differential pumping system

\section{BERYLLIUM WINDOW}

The structure of the beryllium window for the commissioning window system uses the standard window design for the APS wiggler frontend. The oxygen-free-copper (OFHC) water-cooling base is vacuum brazed with the stainless steel flanges and the beryllium foil. A precision survey mark has been scribed on the outside of the flange for use in alignment. The optical aperture of the first beryllium window is $5-\mathrm{mm} \times 5-\mathrm{mm}$ and is $5.5-\mathrm{mm}(\mathrm{V}) \times 8-\mathrm{mm}(\mathrm{H})$ for the second window. 


\section{DISCUSSION}

An accompanying

paper discusses the detailed thermal-mechanical analyses for the commissioning window system ${ }^{10}$. During the first prototype tests, two extending vacuum spool parts will be used to provide the mounting space for a sapphire window viewport. An infrared camera system will be used to measure the surface temperature on filters and windows.

The APS commissioning window has been designed to be a versatile unit as explained below.

For those beamlines, in which only an undulator source is used and low energy $X$-ray photons are required, windowless operation with the differential pumping system is the best setup for maximum photon throughput. Then the same fixed mask in the commissioning window system can be assembled in-between the two ion pumps to control the beam missteering and improve the efficiency of the front-end differential pumping system as shown in Fig. 6. However, many beamlines at the APS have been designed for high energy $X$-ray applications. Then the commissioning window may kept for continued use with the experiments.

Further performance optimization for the APS undulator window system will be developed based on the results of the first prototype test, which is scheduled in the next few months.

\section{ACKNOWLEDGMENTS}

Careful editing by Susan Picologlou is gratefully acknowledged. This work was supported by the U. S. Department of Energy, BES-Material Sciences, under contract No. W-31-109-ENG-38.

${ }^{\text {t}}$ R. J. Dejus, Barry Lai, Elizabeth R. Moog, and Efim Gluskin Undulator A Characteristics and Specifications: Enhanced Capabilities. Argonne National Laboratory Report ANL/APS/TB-17 (1994)

${ }^{2}$ D. Shu and T. M. Kuzay, Nucl. Instrum. and Meth. A 347 (1994) 584-590.

${ }^{3}$ C. Liu, D. Shu, T.M. Kuzay and R. Kersevan, Monte Carlo simulations of the vacuum performance of differential pumps at the Advanced Photon Source, these proceedings

${ }^{4}$ U.S. patent 5, 404,014, Feb 1994

${ }^{5}$ T. M. Kuzay and Z. Wang, "Review of Window and Filter Requirements for Commissioning of the Advanced Photon Source Insertion Device Beamlines", Argonne National Laboratory report LS-236

${ }^{6}$ H. L.T. Nian, T. M. Kuzay and D. Shu, ANL, unpublished information, 1995

'Z. Wang, T. M. Kuzay, D. Shu, ANL. unpublished information, 1995

${ }^{3}$ D. Shu, Y. Li. D. Ryding and T.M. Kuzay, Rev. Sci. Instrum. 66. (1995) 1783-1785

"H. L. Thomas Nian. D. Shu, I.C. Sheng and T.M. Kuzay,

Nucl. Instrum. Meth. A347 (1994) 657-663.
${ }^{10} \mathrm{~T}$. M. Kuzay, H. L. Thomas Nian, Zhibi Wang and D. Shu, Performance analysis of the commissioning filter and window assembly for insertion device beamlines at the Advanced Photon Source, these proceedings
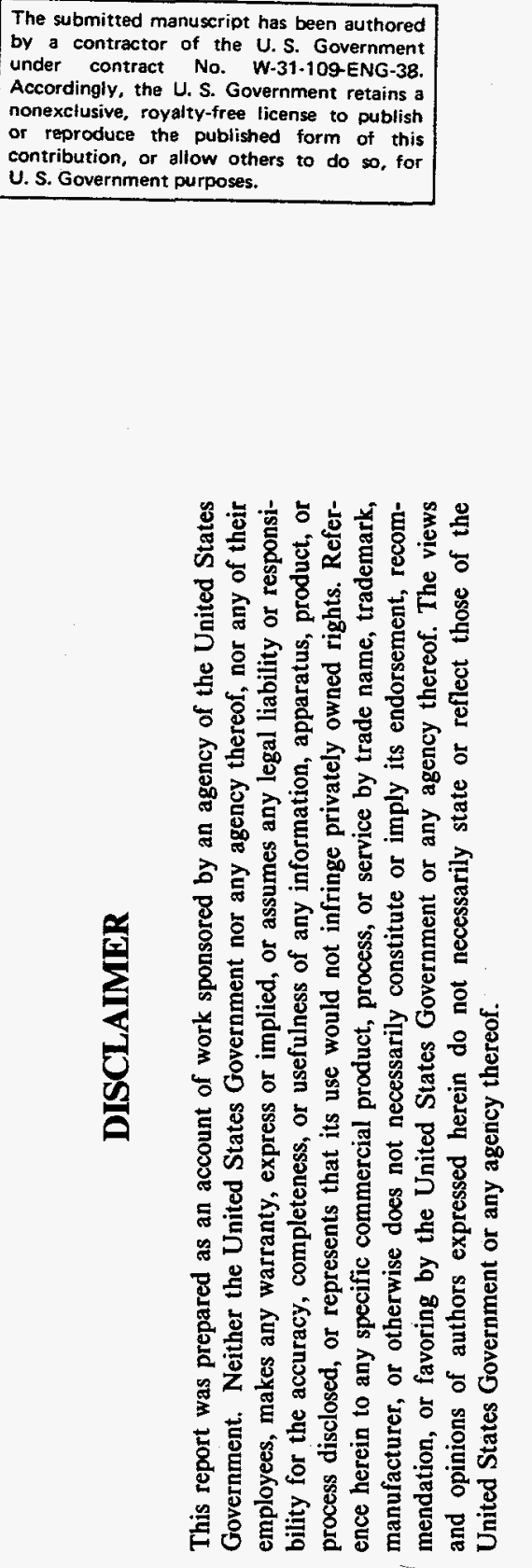


\section{DISCLAMMER}

Portions of this document may be illegible in electronic image products. Images are produced from the best available original document. 